\title{
Nonlinearity Analysis of Quantum Capacitance and its Effect on Nano- Graphene Field Effect Transistor characteristics
}

\section{Declaration}

I am in full confidence conform that for this manuscript we do not any conflict of interest

Munindra, Deva Nand

Electronics and Communication Engineering, Delhi Technological University, Delhi, India

Bawana Road, Shahbad Daulatpur Village, Rohini, Delhi, 110042

\section{About Authors}

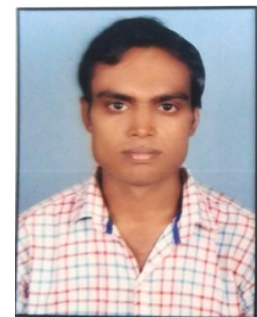

MUNINDRA was born in Roorkee, Uttrakhand-India in August 1990. He received the B. Tech. and M. Tech. degrees in Electronic and Communication engineering from the Uttrakhand Technical University, Dehradun, in 2011 and National Institute of Technology (NIT) Delhi in 2016 respectively. Currently he is pursing the Ph.D. degree in Electronic and Communication engineering from Delhi Technological University, Delhi-India since 2017.

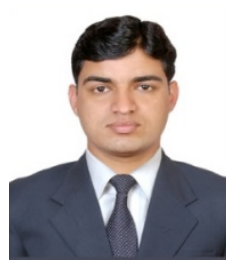

Deva Nand has completed his Ph.D. from Department of Electronics and Communication Engineering, Delhi Technological University, Delhi, India. At present he is an Assistant Professor in Department of ECE, DTU, Delhi, India. A life member of ISTE, member of IAENG and IEEE. His research interests include Microelectronics, Analog VLSI Design. 


\title{
Nonlinearity Analysis of Quantum Capacitance and its Effect on Nano- Graphene Field Effect Transistor characteristics
}

\author{
Munindra and *Deva Nand \\ Department of Electronics and Communication Engineering, Delhi Technological University, Delhi, India \\ kumar.muninder90@gmail.com,*devkamboj07@gmail.com
}

\begin{abstract}
A simple, compact, and fundamental physics-based quasi-analytic model for Single layer graphene field effect transistors (GFETs) with large area graphene is presented in which the quantum mechanical density gradient method is utilised. The basic device physics of the two-dimensional (2D) graphene channel is studied analytically. This modeling leads to the precise drain current calculation of the GFETs. The drain current calculation for GFETs starts from charge carrier concentration, its density of states and quantum capacitance(QC). QC depends on the channel voltage as a function of gate to source voltage $V_{g s}$ and drain to source voltage $V_{\text {ds }}$ primarily. The formulation of the drain current with velocity saturation has been done by the Monte Carlo simulation method. The performance of the analytical GFETs model is present the precise values of QC, its impact on drain current and transfer as well as output characteristics. The impact of QC at nanometer technology adds the nonlinearity to characteristics curves. The proposed method provides better results as compared with the previous analytical and simulated results.
\end{abstract}

Keywords - quasi-analytical model, quantum capacitance, GFETs, nonlinearity

\section{INTRODUCTION}

Heart of the electronic devices is always the ability of a particular material to control its electronic properties in the presence of another electric field. Since the semiconductor industry is already out of scaling limits for silicon-based devices. So, this industry needs the next excellent alternate of silicon. Graphene, after its first physically stable noble experimentation in 2004 is promising to be the next alternate of silicon. Graphene is a single atomic (carbon) thin or thick layer $\left(\sim 3.34 \times 10^{-10} \mathrm{~m}\right)$ so it is called as 2-D material [1-5], along with high charge carrier mobility [2] of value more than $10^{5} \mathrm{~cm}^{2} / \mathrm{V}-\mathrm{sec}$ and even thermodynamically stable. The CVD grown graphene layer and graphene growth over base other then silicon could be a possible option for high frequency and analog electronic devices and circuits [4-5]. The very first physical demonstration of field emission effect in graphene layers comes in the form of an experimental device in 2007 [6]. As the technology advancement is very fast, in few next years interesting GFETs models with the intrinsic transit frequency and the maximum frequency of GFETs operation is comparable and higher than similar size CMOS nanometer technology [7-10]. As the current saturation effect in a single layer [11] and bilayer layer graphene [12], with a high value of transconductance and transistor resistance directly affect the GFETs performance. Gate/channel length of graphene has an impact on the operating frequency of the proposed GFETs model, therefore, besides the high resistance and 
transconductance values, graphene RF circuits find their application in defense in real life, so this is the main focus of this particular work. Other important, compacts and fundamental physics-based quasi-analytic modelling for (GFETs) with large area graphene is also given in literature [13]. Some other work like GFETs modelling based on basic physics explores drain current, capacitance, and charge parameters for graphene channel [14-17]. Explicit compact model of drain current [14] has been extended in [15] to explore AC and transient behaviour of the GFETs. Both of these physical framework is field effect model based on drift-diffusion charge carrier transport mechanism. The Ward-Dutton charge partition scheme is implemented to model the source-drain charge together with transconductance and self-capacitance. These [16-17] compact model is benchmarked with measured current-voltage characteristic with accuracy for prototype devices and predictive behaviour. Also, few comprehensive simples and compact mathematical model provides graphene field effect transistor's physical parameters closed form formula's like $\mathrm{g}_{\mathrm{m}}, \mathrm{r}_{0}, \mathrm{C}_{\mathrm{gs}}$, and $\mathrm{C}_{\mathrm{gd}}$. Many other comprehensive, compact and analytical modeling of GFETs gate capacitance and quantum capacitance as dependent modeling parameter already done. But all of them either are limited to modelling and simulation of GFETs characteristics like some latest GFETs models [18-20], or are complex or are practically absurd [21-23] with no future application in device and circuits. Latest GFETs model is [24].The proposed model is keep its nature lucid mathematically, present the explicit quantum capacitance, drive the drain current formulation using it and also present the nonlinearity of characteristic curves of the GFETs at nanometer technology. Since noise is always a key element to disturb the performance of the FETs and GFETs in real world electronic device and circuits, so the study of nonlinearity to the transfer as well as output characteristics is our addition to the modelling and simulation of the GFETs. Dual gate control along with the nonlinearity study has been given in transfer characteristic curves in result and discussion section. Dual gate control to large area graphene GFETs is also a new concept for the proposed GFET modeling at 300nm technology.

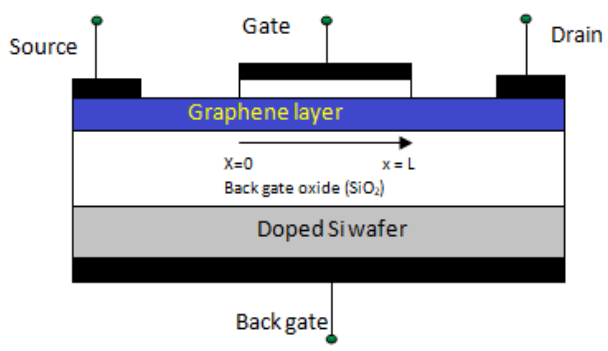

Figure 1 2-D Cross-sectional view of the proposed GFETs.

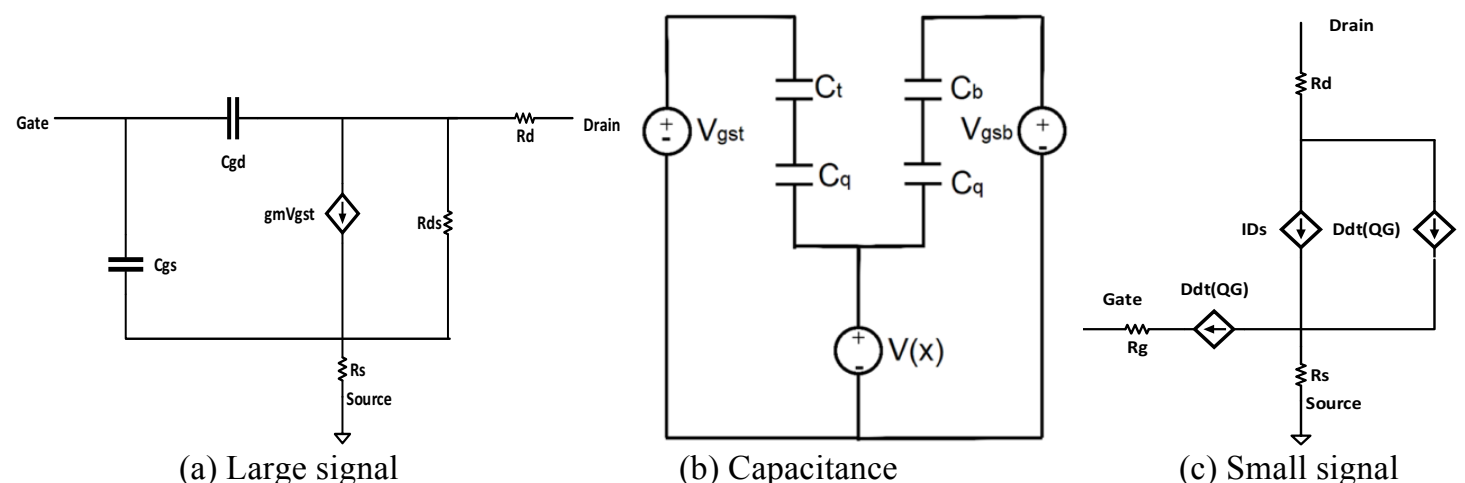

Figure 2 (a), (b), (c) Equivalent circuit of the proposed GFETs (all h-parameter symbols have their usual meaning). 
The structure of this paper is as follows, the detailed explanation of physic-based GFET model starts from charge carrier concentration and density of states in the section II analytical model. Furthermore, section II explains quantum capacitance which leads to the drain current formulation and help to plot the graphical results for the GFET at $1000 \mathrm{~nm}$ and $300 \mathrm{~nm}$ channel length. Where this is noticeable that the source is grounded. Section III discussed and compare the experimental results with the proposed model results. Finally, section IV concludes this GFET model.

\section{Analytical Model}

\section{A. Device structure of GFETs}

The proposed compact model is based on well-established dual-gate MOS structure which is shown in Fig. 1 as a 2-D cross-sectional view of proposed GFETs, which consists two gates (top and back gate), a large area graphene sheet as channel placed on the thick $\mathrm{SiO}_{2}$ back-gate oxide layer (300 $\mathrm{nm}$ and 1000nm), where heavily doped silicon wafer act as a back gate. This article emphasis on p-type GFETs, while a similar approach is applicable to the n-type GFETs also. The $C_{g s}$ and $C_{g d}$ are gate to source and gate to drain capacitance. $R_{d s}, R_{d}$ and $R_{s}$ are the drain to source resistance, drain resistance and source resistance respectively as shown in Fig. 2.(a), all of these parameters nonlinear effect at nanometer technology is considered minimum since these can be control externally by suitable parameter modelling. $\mathrm{V}(\mathrm{x})$ is shown above in Fig. 2 (b) as channel voltage, which is variable and depends on x's value from $0(0)$ to $\mathrm{L}\left(V_{D S}\right)$. While $C_{t}, C_{b}$ and $C_{q}$ are top gate capacitance, back gate capacitance and, quantum capacitance respectively and $V_{g s_{t}}$ and $V_{g s_{b}}$ applied internal voltages. $\mathrm{V}_{\mathrm{ds}}$ and $\mathrm{V}_{\mathrm{BG}}$ are the external applied drain to source and back gate voltage. Small signal equivalent circuit of the proposed GFETs is also shown in Fig.2 (c) where $R_{s}, R_{d}$ and $R_{g}$ are the source, drain and gate resistance respectively.

\section{B. Formulation of charge carrier sheet density $\left(\rho_{g r}\right)$ and quantum capacitance calculation $\left(C_{q}\right)$}

2-D Energy and wave vector relation for the mobile $\pi$ electrons in graphene can be formulated [25] as $E(k)=s h v_{\mathrm{f}}|k|$ while $\mathrm{s}$ is equal to +1 in the conduction band and s equal to -1 in valance band for the first Brillouin Zone (BZ) in graphene layers. Where (h) is the reduced form of Planck's constant and Fermi velocity $\left(v_{\mathrm{f}}\right)$ is of high magnitude equal to $1 \times 10^{8} \mathrm{~cm} / \mathrm{s}$ along with $|k|$ a $2 \mathrm{D} \mathrm{x}-\mathrm{y}$ plane wave vector at the origin $|k|=0$ is referred to "Dirac point." Each Dirac point is double fold spin degeneracy $g_{s}=2$ and contains double valley $g_{v}$ $=2$, thus the number of state in a graphene layer is

$N(E)=g_{s} g_{v} \frac{A k^{2}}{4 \pi\left(\hbar v_{\mathrm{f}}\right)^{2}}$

here A is an area of the particular layer since the linear charge density of states [22] for an intrinsic graphene layer is a differential form of the number of the state of the particular layer with respect to the energy of the particular states, which can be expressed as,

$\rho_{g r}=\frac{1}{A} \frac{d N}{d E}=\frac{2}{\pi} \frac{|E|}{\left(\hbar v_{\mathrm{f}}\right)^{2}}$ 
and so, to calculate the charge carriers (hole) concentration in 2D graphene sheet based on fundamental physics can be formulated as

$p=\int_{-\infty}^{E c v} \rho_{g r}(E)[1-f(E)] d E$

after replacing $\mathrm{f}(\mathrm{E})$ as the Fermi-Dirac distribution function and $\rho_{g r}(E)$ too, the no. of holes in the graphene sheet can be written as follows, where $E c v$ work as reference energy (i.e., it will be taken $E c v=0$ )

$p=\int_{-E c v}^{\infty} \frac{2}{\pi} \frac{|E|}{\left(\hbar v_{\mathrm{f}}\right)^{2}}\left[\frac{1}{\exp \frac{\left(E+E_{F}\right)}{K_{B} T}+1}\right] d E$

similarly, no. of the electron in graphene channel with respect to field dependent charge carrier can be represented as

$n=\int_{0}^{\infty} \frac{2}{\pi} \frac{|E|}{\left(\hbar v_{\mathrm{f}}\right)^{2}}\left[\frac{1}{\exp \frac{\left(E-E_{F}\right)}{K_{B} T}+1}\right] d E$

thus the total charge $\left(\mathrm{Q}_{\text {net }}\right)$ is equal to $(p-n)$ and could be utilized to estimate total charge sheet density $Q_{g r}$, which is equal to $\mathrm{q} *(\mathrm{p}-\mathrm{n})$ as follows

$Q_{g r}=\frac{2}{\pi} \frac{q|E|}{\left(\hbar v_{\mathrm{f}}\right)^{2}} \int_{0}^{\infty}\left[\frac{1}{\exp \frac{\left(E-E_{F}\right)}{K_{B} T}+1}-\frac{1}{\exp \frac{\left(E+E_{F}\right)}{K_{B} T}+1}\right] d E$

the quantum capacitance of the proposed device is $\mathrm{C}_{\mathrm{q}}$ which can be calculated [22] as the differential of the channel sheet charge density $Q_{g r}$ with respect to $V_{g r}$, where $V_{g r}$ is graphene channel voltage

$\mathrm{C}_{\mathrm{q}}=-\frac{\mathrm{d} Q_{g r}}{\mathrm{~d} V_{g r}}$

while minus sign here reflects the impact of more and more positive voltage applied to gate directly increase the positive channel voltage i.e. more negative charge results in the graphene layer.

$\mathrm{C}_{\mathrm{q}}=-\frac{\mathrm{d}}{\mathrm{d} V_{g r}}\left\{\frac{2}{\pi} \frac{q|E|}{(\hbar v f)^{2}} \int_{0}^{\infty}\left[\frac{1}{\exp \frac{\left(E-E_{F}\right)}{K_{B} T}+1}-\frac{1}{\exp \frac{\left(E+E_{F}\right)}{K_{B} T}+1}\right] d E\right\}$

To solve the integral term in the above equation take constants outside and substitute exponential term $\exp \frac{\left(E \pm E_{F}\right)}{K_{B} T} \pm 1$ by $\mathrm{x}$ and $d E$ by $\frac{K_{B} T}{x} d x$ we get simplified equation as 


$$
\mathrm{C}_{\mathrm{q}}=-\frac{\mathrm{d}}{\mathrm{d} V_{g r}}\left(\frac{2}{\pi} \frac{q}{(\hbar v f)^{2}}\right)\left\{\left[\int_{A}^{\infty}\left|K_{B} T \ln x-E_{F}\right| \frac{1}{x} * \frac{K_{B} T}{x}\right] d x-\left[\int_{B}^{\infty}\left|K_{B} T \ln x+E_{F}\right| \frac{1}{x} * \frac{K_{B} T}{x}\right] d x\right\}
$$

a simplified quantum capacitance formulated above in Eqn. (9) can be rewritten as

$$
\mathrm{C}_{\mathrm{q}}=-\frac{\mathrm{d}}{\mathrm{d} V_{g r}}\left(\frac{2}{\pi} \frac{q}{(\hbar v f)^{2}}\right)\left\{\text { part } A^{\prime}+\operatorname{Part} B^{\prime}\right\}
$$

by dividing integral into two parts and naming as part $A^{\prime}$ and $P$ art $B^{\prime}$ as

$$
\begin{aligned}
& \int_{A}^{\infty}\left|K_{B} T \ln x-E_{F}\right| \frac{1}{x} * \frac{K_{B} T}{x} d x \\
& \int_{B}^{\infty}\left|K_{B} T \ln x+E_{F}\right| \frac{1}{x} * \frac{K_{B} T}{x} d x
\end{aligned}
$$

where $A$ is $\exp \left(\mathrm{E}_{\mathrm{F}} / \mathrm{K}_{\mathrm{B}} \mathrm{T}+1\right)$ and $\mathrm{B}$ is $\exp \left(-\left(\mathrm{E}_{\mathrm{F}} / \mathrm{K}_{\mathrm{B}} \mathrm{T}+1\right)\right)$, so after putting limits in exponential term and replacing $\mathrm{x}=0$ and by solving Eqn. (11) and (12) by two-part integral substitution method we get combined solution for both part $A^{\prime}$ and Part $B^{\prime}$ and further depends on positive and negative sign as given in Eqn. (13), where $K_{B} T \ln x \pm E_{F}$ as part I and $\frac{1}{x} * \frac{K_{B} T}{x}$ as part II,

$\int_{A, B}^{\infty}\left|K_{B} T \ln x \pm E_{F}\right| * \frac{1}{x} * \frac{K_{B} T}{x} d x$

Now assuming $E_{F}$ as constant since $E_{F}=q \cdot V_{c h}$ and by following standard mathematical rules to solve this integral and after applying limits we get solved part as given below

$\frac{\left(K_{B} T\right)^{2}}{A}(\ln A+1)-E_{F} \frac{\left(K_{B} T\right)}{A}$

similarly, after solving part II

$\frac{\left(K_{B} T\right)^{2}}{B}(\ln B+1)+E_{F} \frac{\left(K_{B} T\right)}{B}$

Now by sum-up part $A^{\prime}$ and Part $B^{\prime}$, one could represent a solved integral in quantum capacitance as follows

$$
\mathrm{C}_{\mathrm{q}}=-\frac{\mathrm{d}}{\mathrm{d} v_{g r}}\left(\frac{2}{\pi} \frac{q}{(\hbar v f)^{2}}\right)\left\{\left[\frac{\left(K_{B} T\right)^{2}}{A}(\ln A+1)-E_{F} \frac{\left(K_{B} T\right)}{A}\right]-\left[\frac{\left(K_{B} T\right)^{2}}{B}(\ln B+1)+E_{F} \frac{\left(K_{B} T\right)}{B}\right]\right\}
$$

By substituting the value of $\mathrm{A}$ and $\mathrm{B}$, and after performing some basic algebraic mathematics, the quantum capacitance can be represented in a simple formula as given below

$$
\mathrm{C}_{\mathrm{q}}=-\frac{\mathrm{d}}{\mathrm{d} v_{g r}}\left(\frac{2}{\pi} \frac{q}{(\hbar v f)^{2}}\right)\left\{\left[\frac{\left(K_{B} T\right)^{2}}{\exp \frac{\left(E_{F}\right)}{K_{B} T}+1}-\frac{\left(K_{B} T\right)^{2}}{\exp \frac{\left(-E_{F}\right)}{K_{B} T}+1}\right]\right\}
$$


By making further math's effort to the above exponential terms and replacing by the hyperbolic trigonometric formulas quantum capacitance could be present as

$$
\mathrm{C}_{\mathrm{q}}=-\frac{2 q}{\pi} \frac{\left(K_{B} T\right)^{2}}{(\hbar v f)^{2}} \frac{d}{d V g r}\left[\frac{2 \sinh \frac{q V g r}{K_{B} T}}{2\left(1+\cosh \frac{q V g r}{K_{B} T}\right)}\right]
$$

By using reverse engineering, we can write quantum capacitance [18] even more simplified as given in $\log$ term

$$
\mathrm{C}_{\mathrm{q}}=-\frac{2 q}{\pi} \frac{q V g r K_{B} T}{(\hbar v f)^{2}} \ln \left[2\left(1+\cosh \frac{q V g r}{K_{B} T}\right)\right]
$$

For the $q V_{g r} \gg K_{B} T$ condition, Eqn. (19) will be simplified to the following single term formula

$$
\mathrm{C}_{\mathrm{q}}=-\frac{2 q^{2}}{\pi} \frac{q|V g r|}{(\hbar v f)^{2}}
$$

As like total charge density in Eqn. (6) has been used to formulate quantum capacitance accurately, channel voltage $V_{g r}$ and quantum capacitance $\mathrm{C}_{\mathrm{q}}$ have useful significance to formulate $\rho_{g r}$. So, that by using basic mathematics technique of two equations and two variable drain current can be formulated by using $\rho_{g r}$.

$$
\begin{aligned}
& Q_{g r}=-\int \mathrm{C}_{\mathrm{q}} * d V_{g r}=-\frac{1}{2} \mathrm{C}_{\mathrm{q}} V_{g r} \\
& \rho_{g r}=\frac{Q_{g r}}{q}=\left|\frac{-\frac{1}{2} C q V_{g r}}{q}\right|
\end{aligned}
$$

while channel voltage $V_{g r}$ directly can be calculated by using basic technique KVL and KCL form the given Fig. 2 (c) and can be expressed as

$V_{g r}=\frac{1}{\mathrm{C}_{\mathrm{t}}+\mathrm{C}_{\mathrm{b}}+0.5 \mathrm{C}_{\mathrm{q}}}\left\{\left[V_{g s_{t}}-V(x)\right] C_{t}-\left[V_{g s_{b}}-V(x)\right] C_{b}\right\}$

The pre-factor of $\frac{1}{2}$ with $C_{q}$ is because of its dependency on $V_{g r}$ and now by putting Eqn. (23) in Eqn. (22) we get a simple a second order algebraic Eqn.

$C_{t}^{2}+\left(C_{t}+C_{b}\right) C_{q}-\left[V_{g s_{t}}-V(x)\right] C_{t}-\left[V_{g s_{b}}-V(x)\right] C_{b}=0$

Thus by rearrange the equation we get a quadratic equation of $C_{q}$ in terms of $V_{g s_{t}}, V_{g s_{b}}$ and $V(x)$. Where $V(x)$ is the channel voltage depend on $\mathrm{x}(0$ to $\mathrm{L})$ and the value of $V(x)$ of at $(\mathrm{x}=$ $0)$ is $0 \mathrm{~V}$, while at $(\mathrm{x}=\mathrm{L})$ is $V_{d s} \mathrm{~V}$.

\section{Drain current calculation}

For drain current calculation at very first we need to focus on charge charier velocity $v=v(x)$ mainly through the channel ( $\mathrm{x}=0$ to $\mathrm{L}$ ) from (1). A low saturation velocity characteristic is shown by Monte Carlo simulation [26] at steady state is 
$v=\frac{\mu E}{1+\frac{\mu|E|}{v_{s a t}}}$

Where E represents the electric field, $\mu$ is the carrier mobility and $v_{\text {sat }}$ is saturation velocity at low field. The carrier velocity, especially $v_{\text {sat }}$ of graphene channel at the $\mathrm{SiO}_{2}$ platform is strongly affected by rare interfacial scattering of phonons. Experimental work has been talked about the two dominated optical phonons at $\mathrm{SiO}_{2}$ and graphene interface. They have been shown a magnitude of $59 \mathrm{meV}$ and $155 \mathrm{meV}$ respectively but one can describe this phenomenon by a single phonon only with enough accuracy and with the energy of $\hbar \Omega$. By the approach used [27] to calculate $v_{s a t}, v_{\text {sat }}$ is depends on charge carrier sheet density $\left(\rho_{s}\right)$ and the phonons effective energy $\Omega$.

$v_{\text {sat }}=\frac{\Omega}{\sqrt{\pi \rho_{g r}}}$

Charge carrier-interaction phenomenon in graphene-MOSFET channel introduces a new term as $0.5+A . V^{2}(x)$ [26] to correct the calculation of $v_{\text {sat }}$ to one decimal point at the channel drain end. Now Eqn. (26) will become as,

$v_{s a t}=\frac{\Omega}{\left(\pi \rho_{g r}\right)^{0.5+A . V^{2}(x)}}$

here $\mathrm{A}$ is a dimensionless important factor of order $10^{-3}$, when $\rho_{S}$ is of magnitude $10^{12} \mathrm{~cm}^{-2}$ and the final unit will be $\mathrm{cm} / \mathrm{s}$. Now using $\mathrm{E}=\left(-\frac{d V}{d x}\right)$ and Eqn. (20) drain current $\left(\mathrm{I}_{\mathrm{d}}\right)$ becomes

$I_{d}=-q \rho_{g r} \frac{\mu\left(-\frac{d V}{d x}\right)}{1+\frac{\mu\left(-\frac{d V}{d x}\right)}{v_{s a t}}} W$

after integrating left side 0 to $\mathrm{x}$ and $0 \mathrm{~V}$ to $V_{d s} \mathrm{~V}$ right side, since $V=\mathrm{V}(x)$, is a function of the channel length and by changing the variable of separation $I_{d}$ becomes

$$
\begin{aligned}
& \mathrm{I}_{\mathrm{d}}=\mathrm{q} \mu W \frac{\mu \int_{0}^{V} V^{V} \rho_{g r} d V}{L-\mu \int_{0}^{V} \frac{V}{V_{s} \frac{1}{v_{s a t}} d V}} \\
& \mathrm{I}_{\mathrm{d}}=-\frac{\mu W}{2 L_{e f f}} * \frac{C_{t}}{\mathrm{C}_{\mathrm{t}}+\mathrm{C}_{\mathrm{b}}+0.5 \mathrm{C}_{\mathrm{q}}}\left\{\left(V_{g s}-V(x) / 2\right)-\left(V_{g s_{b}}-V(x) / 2\right)\right\} \\
& L_{e f f}=L-\frac{\mu}{\Omega}\left\{\left[\log \left(\frac{\pi C_{q}}{2 q}\right)+\log \left[\left(V_{g s}-V_{d s}\right) C_{t}+\left(V_{g s_{b}}-V_{d s}\right) C_{b}\right]\right] *\left[0.5 V_{d s}+\frac{A V_{d s}{ }^{3}}{3}\right]+\right. \\
& \int_{0}^{V_{d s}}\left\{\left(\frac{2 q}{\pi C_{q}}\right)+\frac{\left(C_{t}+C_{b}\right)}{\left[\left(V_{g s_{t}}-V_{d s}\right) C_{t}+\left(V_{g s_{b}}-V_{d s}\right) C_{b}\right]}\right\} *\left[0.5 V_{d s}+\frac{A V_{d s}{ }^{3}}{3}\right]
\end{aligned}
$$

\section{RESULTS AND DisCUSSION}

To investigate the quantum capacitance effect on gate capacitance, a basic MOS structure without the back gate of oxide thickness $(300 \mathrm{~nm})$ and top gate oxide of the thickness $(3 \mathrm{~nm}$,), zero voltage as $V_{g s_{t}}, V_{g s_{b}}$ and $V_{D s}$ is arranged for the proposed GFETs model. Quantum 
capacitance of the amplitude of the $10^{\text {th }}$ multiple micron is enhanced overall gate capacitance with respect to the relation $\mathrm{C}_{\mathrm{q}}=\mathrm{C}_{\mathrm{ox}} * \mathrm{C}_{\mathrm{q}} /\left(\mathrm{C}_{\mathrm{q}}+\mathrm{C}_{\mathrm{ox}}\right)$.

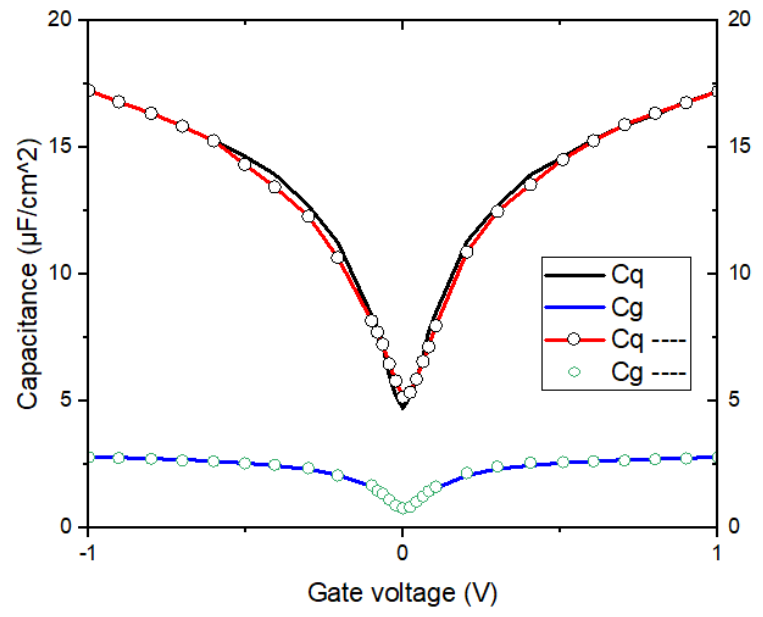

Figure 3 Quantum capacitance effect on overall gate capacitance vs gate voltage. Line graph is simulated proposed model results, while symbolic results graph are analytical results and this is same for all presented result graphs. One of the important aspects of nanometer technology GFETs is that its quantum capacitance plays an important role in terms of device characteristics. Fig. 3 elaborate graphically how the quantum capacitance has an impact on the total gate capacitance at $300 \mathrm{~nm}$ channel length. The proposed model provides better results analytical (with line) and the simulated (with symbol) as compared with [11] for QC as well as gate capacitance at $300 \mathrm{~nm}$. Table 1 explains the numerical values of all important parameters and dimensions of the GFETs model.

Table 1 Dimensions and parameters of the modeled GFETs devices

\begin{tabular}{|l|l|l|}
\hline Parameter & GFET 1 [11] & $\begin{array}{l}\text { GFET } \\
\text { 300nm(proposed) }\end{array}$ \\
\hline $\mathbf{L}(\boldsymbol{\mu m})$ & 1 & .3 \\
\hline $\mathbf{W}(\boldsymbol{\mu m})$ & 2.1 & 2.1 \\
\hline $\mathbf{t}_{\text {ox }}(\mathbf{n m})$ & 15 & 15 \\
\hline $\mathbf{t}_{\text {box }}(\mathbf{n m})$ & 285 & 285 \\
\hline $\mathbf{V}_{\text {gs-top }}(\mathbf{V})$ & 1.45 & .95 \\
\hline $\mathbf{V}_{\text {gs-back }}(\mathbf{V})$ & 2.7 & 2.7 \\
\hline $\mathbf{p}$ & $1.5 * 10^{12}$ & $3 * 10^{12}$ \\
\hline $\boldsymbol{\mu}_{\mathbf{n}}\left(\mathbf{c m}^{\mathbf{2}} / \mathbf{V . s}\right)$ & 1500 & 2500 \\
\hline $\boldsymbol{\mu}_{\mathbf{p}}\left(\mathbf{c m}^{\mathbf{2}} / \mathbf{V . s}\right)$ & 1500 & 2500 \\
\hline $\mathbf{H}(\mathbf{m e V})$ & 56 & 99 \\
\hline $\boldsymbol{\lambda}(\mathbf{n m})$ & $10-100$ & $100-300$ \\
\hline
\end{tabular}

Fig. 4 and Fig. 5 have been explained graphically the transfer characteristic and output characteristic of the proposed GFETs model and compare it with the experimental work [11] mentioned as GFET1 in table 1. GFET1 featuring a high dielectric 2D gate material $\mathrm{HfO}_{2}$ on exfoliated graphene channel and $\mathrm{SiO}_{2}$ is the back-gate dielectric. The output characteristic of the proposed model in Fig. 5, has been shown coherence with the [11], with the applied voltage at source $(0 \mathrm{~V})$, negative drain voltage ( -2 to $0 \mathrm{~V}$ ), top-gate (sweep from -0.75 to $0.75 \mathrm{~V}$ ) as it 
can easily view in a graph with the back-gate voltage of $-40 \mathrm{~V}$. In the same manner transfer characteristic of the GFETs both analytical and simulated is compared with [11] in Fig. 5.

The nonlinearity characteristic behavior of the GFET at 300nm channel has been shown in Fig. 4,5 and 6. Dirac-point shift has been shown in Fig.4 after applying external gate to source voltage for single layer graphene, while for the conventional MOSFETs Dirac point has same contact point of Conduction and valance band. This Dirac-point shift in transfer characteristic and kink presented in the output characteristics is a proof of nonlinear behavior of the GFETs. This nonlinear behavior is tending to enhanced when the channel length of the GFETs is being decreased from $1000 \mathrm{~nm}$ to $100 \mathrm{~nm}$ and below that value as this is given in the latest accepted paper [24]. The dual gate control has been graphically present in the Fig. 6 at same time nonlinear behavior of the GFETs can be observe very easily from Fig 6. e and f. Here we concentrate on the nonlinearity of the transfer characteristics of the Proposed model. A flat line drain current has been shown in the fig. 6.e for -0.1 to $0.1 \mathrm{~V}$ gate to source voltage and beyond this it has shown gradual increment, which is normal in electronic device characteristic curves but a flat drain current shows a nonlinearity in the transfer characteristic curve.



Figure 4 Transfer characteristic of the proposed model i.e. drain current vs top-gate voltage for the proposed GFETs model.
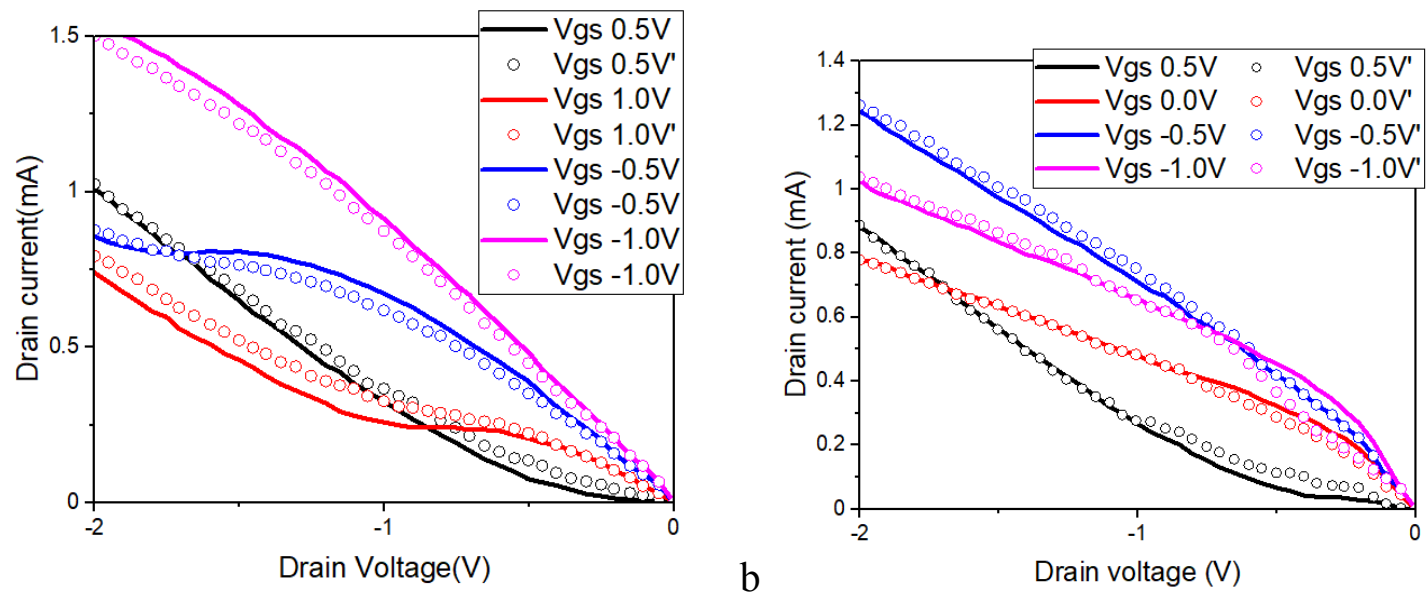

Figure 5.a and 5.b: Output characteristic of the proposed model i.e. drain current vs drain to source voltage for the GFETs model at $1000 \mathrm{~nm}$ in $5 . \mathrm{a}$ and $300 \mathrm{~nm}$ in 5.b. 

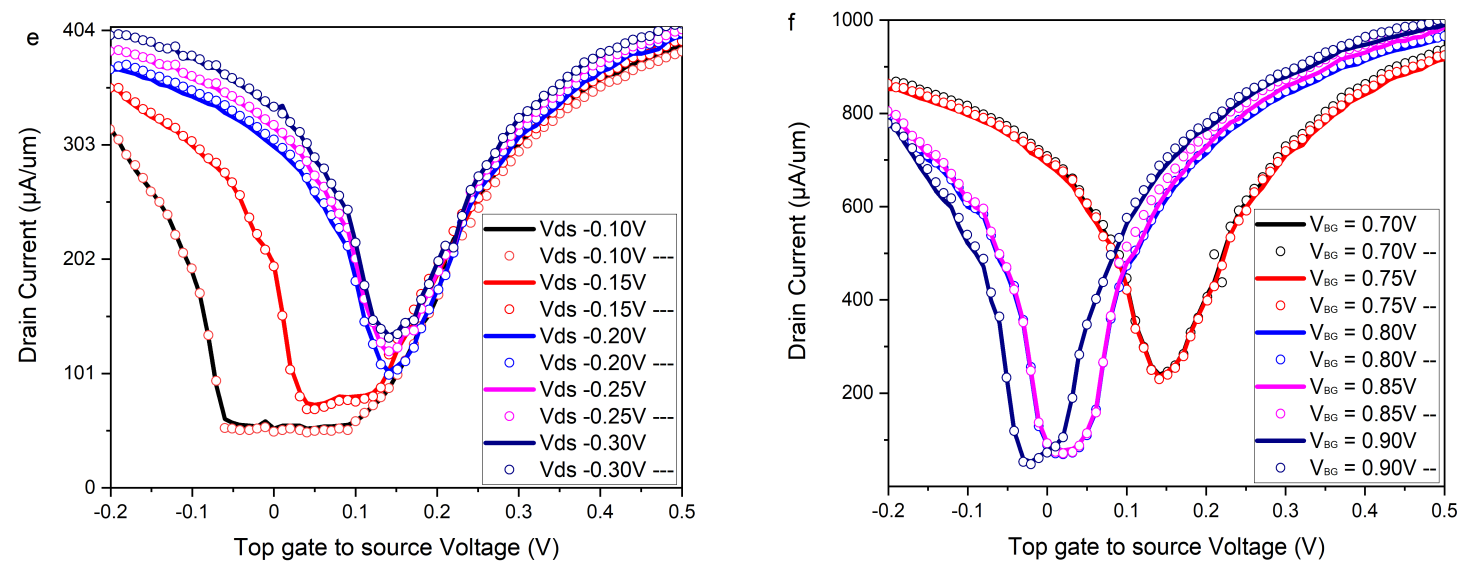

Figure 6.e and 6.f: Transfer characteristic of the proposed model i.e. drain current vs drain to source voltage for the GFETs model. Top gate control by $\mathrm{V}_{\mathrm{ds}}$ and back gate control by $\mathrm{V}_{\mathrm{BG}}$ over drain current is presented in this graph (while for this dual gate control an exception is considered that the graphene sheet is not a large area sheet). $\mathrm{V}_{\mathrm{ds}}$ and $\mathrm{V}_{\mathrm{BG}}$ are the external applied drain to source and back gate voltage.

\section{Conclusion}

A quasi-analytic model for single layer GFETs with large area graphene is presented. Drain current formulation with velocity saturation effect and role of quantum capacitance over the total capacitance is explained with the precise output and transfer characteristics of the proposed GFET model, which facilitate the readers a simple mathematical GFET model. The effect of enhanced quantum capacitance value at nanometer technology adds nonlinearity to the transfer and output drain characteristics of the proposed GFETs model has been presented. Thus the modeling and simulation of GFETs model has present and compare the results with the well established experimental work and it will surly befits the reader to understand basic physic of the GFETs.

\section{References}

[1] K. S. Novoselov, A. K. Geim, S. V. Morozov, D. Jiang, and S. V. Dubones, I. V. Grigorieva and A.A. Firsov "Electric Field Effect in Atomically Thin Carbon Films," Sci. Report, vol. 306, no. 5696, pp. 666-669, 2004.

[2] K. S. Novoselov and A. K. Geim, "The rise of graphene," Nat. Mater., vol. 6, no. 3, pp. 183-191, 2007.

[3] F. Schwierz, "Graphene transistors," Nat. Nanotechnol., vol. 5, no. 7, pp. 487-496, 2010.

[4] A. K. Geim, "Graphene: status and prospects" Science, vol. 324, no. 5934, pp. 15301534, 2009.

[5] K. S. Novoselov, V. I. Falko, L. Colombo, P. R. Gellert, M. G. Schwab and K. kim "A roadmap for graphene," Nature, vol. 490, no. 11, pp. 192-200, 2012.

[6] M. C. Lemme, T. J. Echtermeyer, M. Baus, and H. Kurz, "A graphene field-effect device," IEEE Electron Device Lett., vol. 28, no. 4, pp. 282-284, 2007.

[7] S. J. Han, K. A. Jenkins, A. Valdes Garcia, A. D. Franklin, A. A. Bol, and W. Haensch, "High-frequency graphene voltage amplifier," Nano Lett., vol. 11, no. 9, pp. 3690-3693, 2011.

[8] Y. Wu, D. Farmer, A. Valdes-Garcia, W. Zhu, K. Jenkins, C. Dinmitrakopoulos, et al. “ Record High-frequency graphene voltage amplifier," IEEE IEDM., vol. 11, no. 11, pp. 528-530, 2011.

[9] Y. M. Lin, D. B. Farmer, K. A. Jenkins Y. Wu., J. L.Tedesco, et al., "Enhanced Performance in epitaxial graphene FETs with optimized channel morphology," IEEE 
Electron Device Lett., vol. 32, no. 10, pp. 1343-1345, 2011.

[10] L. Liao, Y. C. Lin, M. Bao, R. Cheng, J. Bai, Y. Liu, et al. "High-speed graphene transistors with a self-aligned nanowire gate," Nature, vol. 467, no. 9, pp. 305-308, 2010.

[11] I. Meric, M. Y. Han, A. F. Young, B. Ozyilmaz, P. Kim, and K. L. Shepard, "Current saturation in zero-bandgap, top-gated graphene field-effect transistors," Nat. Nanotechnol., vol. 3, no. 11, pp. 654-659, 2008.

[12] B. N. Szafranek, G. Fiori, D. Schall, D. Neumaier, and H. Kurz, "Current Saturation and Voltage Gain in Bilayer Graphene Field Effect Transistors," vol. 12, no. 3, pp. 1324$1328,2012$.

[13] S. A. Thiele, J. A. Schaefer, and F. Schwierz, "Modeling of graphene metal-oxidesemiconductor field-effect transistors with gapless large-area graphene channels," $J$. Appl. Phys., vol. 107, no. 9, pp. 094505-1-8, 2010.

[14] D. Jiménez and O. Moldovan, "Explicit drain-current model of graphene field-effect transistors targeting analog and radio-frequency applications," IEEE Trans. Electron Devices, vol. 58, no. 11, pp. 4049-4052, 2011.

[15] D. Jiménez, "Explicit drain-current, Charge and Capacitance model of graphene fieldeffect transistors," IEEE Trans. Electron Devices, vol. 58, no. 12, pp. 4377-4383, 2011.

[16] I. Z. Gennady, A. T. Alexander, K. B. Daria and V. M. Evgeny "Small-Signal Capacitance and Current parameter Modeling in Large-Scale Hgh-Frequency Graphene Field Effect Transistors," IEEE Trans. Electron Devices, vol. 60, no. 6, pp. 1799-1806, 2013.

[17] J. D. A. Morales, S. Frégonèse, C. Mukherjee, C. Maneux and T. Zimmer, "An Accurate physics-based Compact Model for Dual-gate Bilayer Graphene FETs," IEEE Trans. Electron Devices, vol. 62, no. 12, pp. 4333-4339, 2015.

[18] Abhishek Kumar Upadhay, Ajay kumar Kuashwaha and Santosh Kumar Vishvakarma "A Unified Scalable Quasi-Ballistic Transport Model of GFET for circuit Simulations," IEEE Trans. Electron Devices, vol. 65, no. 2, pp. 739-746, 2018.

[19] Abhishek Kumar Upadhay, Ajay kumar Kuashwaha, Priyank Rastogi, Yogesh Singh Chauhan and Santosh Kumar Vishvakarma "Explicit Model of Channel Chagre, Backscattering, and Mobility for Graphene FET in Quasi-Ballistic Regime," IEEE Trans. Electron Devices, vol. 65, no. 10, pp. 739-746, 2018.

[20] Nagothu Karmel Kranthi, Abhishek Mishra, Adil Meersha, and Mayank Shrivastava, "On the ESD Behavior of Large-Area CVD Graphene Transistors: Physical Insights and Technology Implications ," IEEE Trans. Electron Devices, vol. 66, no.1, pp. 743-751, 2019.

[21] F. Pasadas, and D. Jimenez, "Large-signal Model of Graphene Field Effect TransistorPart I: Compact modeling of GFET Intrinsic Capacitance," IEEE Trans. Electron Device, vol. 63, no. 7, pp. 2936-2941, 2016.

[22] F. Pasadas, and D. Jimenez, "Large-signal Model of Graphene Field Effect TransistorPart II: Circuit Performance Benchmarking," IEEE Trans. Electron Device, vol. 63, no. 7, pp. 2942-2947, 2016.

[23] J. J. Ebers and J. L. Moll, "Large-Signal behavior of junction transistors," Proc. IRE, vol. 42, no. 12, pp. 1761-1772, 1954.

[24] Munindra and Deva Nand, "Nonlinearity and scaling trends of quasiballistic graphene field-effect transistors targeting RF applications," Journal of Coputational Electronics, 10.1007/s10825-021-01772-x, Aug 2021

[25] T. Fang, A. Konar, H. Xing, and D. Jena, "Carrier statistics and quantum capacitance of graphene sheets and ribbons," Applied Physic Letters, Vol. 91, no.092109-1-8, 2007.

[26] A. Akturk and N. Goldsmen, "Electron transport and full-band electron-phonon interactions in graphene," Journal of Applied Physics, Vol. 103, no. 0537021-1-7, 2008. 
[27] I. Meric, N. Baklitskaya, P. Kim and K. L. Shepard, "RF performance of top-gated, zerobandgap graphene field-effect transistors," IEDM, Vol. 21, no. 2, pp.15-18 2008. 